Re-pensando

una pedagogía

para la paz desde

la Universidad,

(Reflexiones)

Dr. José Miguel Ayerdis García. 


\section{Re-pensando una pedagogía para la paz desde la Universidad, (Reflexiones)}

Re-thinking a pedagogy for peace from the University, (Reflections)
Dr. José Miguel Ayerdis García.

Dr. En ciencias históricas, catedrático universitario, autor de distintos artículos científicos en revistas nacionales e internacionales, actualmente Director de la Dirección de Relaciones Internacionales de la UNAN-Managua. https://orcid.org/0000-0001-9677-7786 mayermis@unan.edu.ni

Recibido: 10 Mayo 2019 Aceptado: 05 de Junio 2019

(C) (9) Copyright $\odot 2019$ UNAN-Managua (c)

\section{RESUMEN}

Este trabajo es un aporte que los académicos estamos haciendo desde la Universidad para el fomento de una cultura de paz y convivencia que, desde el 2018 está en agenda como consecuencia de la crisis política en la que se vio inmersa la sociedad nicaragüense. Esta propuesta problematiza acerca del lugar y el papel del docente universitario en la búsqueda de prácticas discursivas que canalicen las diferencias y/o contradicciones por cauces cívicos, sabiendo que la sociedad en general quiere paz para continuar mejorando sus condiciones de vida material y espiritual. Lo que pretende esta investigación, es responder a preguntas como, ¿estamos trabajando de verdad en una agenda individual y/o colectiva en función del fortalecimiento del liderazgo docente y contribuyendo de manera concreta a la solución de los problemas que enfrenta la sociedad nicaragüense a consecuencia de la crisis política iniciada el pasado 18 de abril? ¿Creemos en los valores de una cultura de paz (ya institucionalizada por medio del decreto de ley 985) como convergencia para el fortalecimiento del bien común? Por último, ¿estamos desarrollando estrategias pedagógicas en las aulas para el fomento de valores que contribuyan a una visión positiva, constructiva-propositiva y tolerante en los estudiantes, buscando contrarrestar los discursos destructivos que, las burbujas mediáticas, a diario inoculan en las mentes de los jóvenes?

\section{ABSTRACT}

This work is a contribution that as academics we are making from the University on the theme of promoting a culture of peace and coexistence, since 2018 on the agenda as a result of the political crisis that was immersed in Nicaraguan society. This proposal problematizes the place and role of university professors in the search for discursive practices that channel differences and/or contradictions through civic channels, knowing that society in general wants peace in order to continue improving their material and spiritual living conditions. The purpose of this research is to answer questions such as, are we really working on an individual and/or collective agenda in terms of strengthening teacher leadership and contributing in a concrete manner to the solution of the problems facing Nicaraguan society as a result of the political crisis that began on April 18? Do we believe in the values of a culture of peace (already institutionalized through Decree-Law

1 Dejo constancia que lo expresado en este artículo es única y exclusiva responsabilidad de su autor. Son puntos de vista sustentados en la interacción diaria con los diferentes grupos y/o componentes de nuestra comunidad universitaria. Mi cariño y respeto para nuestras autoridades superiores, docentes y trabajadores administrativos, quienes trabajan con mucha dedicación por la calidad y pertinencia de la educación superior, fortaleciendo de esta manera el liderazgo de nuestra Universidad en el país. 
985) as a convergence for the strengthening of the common good? Finally, are we developing pedagogical strategies in the classrooms for the promotion of values that contribute to a positive, constructive-positive and tolerant vision in the students, seeking to counteract the destructive discourses that the media bubbles inoculate in the minds of young people on a daily basis?

\section{Introducción}

os dramáticos acontecimientos políticos durante el mes de abril del año 2018, cuyas - secuelas aún estamos viviendo en el país, han puesto en agenda un tema de discusión postergado, o en el mejor de los casos no abordado de manera sistemática y a profundidad en la Universidad Nacional Autónoma de Nicaragua, UNAN-Managua. Nos referimos a la relación entre el bien común, como concepto integral de comunidad y su correlato moral, entendido como cultivo de virtudes asociadas a una visión positiva y estable entre los diferentes componentes de la comunidad universitaria y la sociedad.

Desde hace más de una década, el gobierno de Nicaragua viene haciendo ingentes esfuerzos por la mejora de la vida material de todos sus habitantes, sin discriminación alguna. Para realizarlo, ha tenido que sortear infinidad de trabas (presiones externas) que el modelo capitalista (y los poderes que los mueven) imponen. Esfuerzos que buscan la construcción de un modelo propio, con márgenes de autonomía, visión colectiva y el cultivo de valores que enfrenten el neoliberalismo, cuyas expresiones se manifiestan, además de la alienación colectiva, en el control de las fuentes de financiamiento, las amenazas, el chantajes y abyección de grupos económicos locales a los poderes centrales, e imperiales.

De igual manera, en la actualidad se vive un contexto internacional complejo, donde se enfrentan por un lado, posiciones supremacistas unipolares, defendida por los halcones que dirigen la política norteamericana y sus financiadores, las empresas armamentísticas y grandes trust industriales o grupos financieros; por el otro, visiones multipolares asumidas como principio, por la mayor parte del concierto de naciones del planeta que desean un mundo en paz. A ello se suma, el ambiente de descontrol generado por la enorme maraña de redes sociales y conglomerados audiovisuales tradicionales y transnacionales, que producen mensajes y noticias manipuladas de manera permanente y sin descanso, obstaculizando el establecimiento de un consenso sólido entre los pueblos y sociedades deseosas de construir un mundo distinto, amigable con el medio ambiente, superando los egoísmos, sectarismos, complejos racistas y discriminatorios.

La resignación a un "estatus quo" político y económico en crisis (el capitalismo), es el punto de vista que se quiere imponer por medio de mensajes que nos llegan a diario, de diferentes formas y canales de difusión masiva. Los prejuicios que sustentaban el discurso de la guerra fría, de comunismo, sinónimo de ausencia de libertad, de peligros a los derechos a acumular capital (bajo el pretexto de la libertad individual) y a la explotación sin freno (la cantaleta del libre mercado), han vuelto en las últimas décadas, en apariencia "renovados", tutelados o promovidos por grupos heterogéneos, religiosos, profesionales, intelectuales, feministas de nuevo cuño, y movimientos sociales de ONG. Grupos oscurantistas que de manera violenta (o por medio de un discurso de fachada crítica al modelo hegemónico del imperialismo), se oponen a cualquier proyecto de gobierno o estado, mínimamente novedoso o progresista, convirtiéndose en los defensores más activos del modelo capitalista. 
Frente a prácticas discursivas seudo avanzadas, huecas en su esencia, sustentadas en teorías y hechos históricos provenientes de modelos hegemónicos, eurocéntricos que satanizan todo proyecto sustentado en nuestras vivencias históricas y culturales, las universidades públicas, de mayor arraigo, presencia y autoridad, deben establecer una agenda nacional que ponga en perspectiva el lugar que ocupa la academia en la búsqueda de consenso. Ir más allá de la superación de la crisis de abril, como se sabe, generada por factores políticos violentos en contubernio con ONGs, empresarios inescrupulosos y religiosos extremistas. Ser promotores (como ya se vienen haciendo desde la UNAN-Managua) de canales o relaciones estables, permanentes y de respeto con los distintos componentes que conforman la sociedad nicaragüense en general, sobre todo, trabajando en la construcción de alianzas estratégicas con sectores vulnerables de la sociedad, grupos sociales, indígenas, iniciativas culturales y emprendimientos económicos, en esencia creyentes de la utopía "que es posible un mundo mejor".

En este sentido, el docente es un actor importante dentro de la comunidad universitaria y la sociedad en general, orientando y facilitando procesos de convivencia, desde las aulas de clase, en la interacción diaria con estudiantes, claustro, amigos, familiares. En cualquier espacio social donde se encuentre, se espera que establezca pautas o formas para la comprensión y/o aprehensión de modelos sociales y culturales, sean estos progresistas o revolucionarios. Su condición académica le habilita y exigen la difusión y/o socialización de la cultura institucional, por medio de su presencia, de manera sistemática, beligerante y constructiva, en eventos, redes académicas culturales y sociales, aprovechando la movilidad e intercambio nacional e internacional.

La posición "privilegiada" del docente dentro de la comunidad académica nacional, le permite promocionar reglas de convivencia, respeto a las diferencias de ideas; estructurar un pensamiento crítico a partir del contrastaste o puesta en cuestión del origen e intención de las fuentes y de los contenidos de los mensajes de la información que se consume a diario. No es gratuito el cognomento de guía que se le adjudica al docente, lo que significa que debe dejar constancia de su liderazgo sin fisura, en el aula, entorno afectivo inmediato y comunitario.

Lo anterior nos lleva a preguntarnos, si estamos trabajando de verdad en una agenda individual y/o colectiva en función del fortalecimiento del liderazgo docente y contribuyendo de manera concreta a la solución de los problemas que enfrenta la sociedad nicaragüense, a consecuencia de la crisis política iniciada el pasado 18 de abril de 2018.

De igual manera, si creemos en los valores de una cultura de paz (ya institucionalizada por medio del decreto de ley 985) como convergencia para el fortalecimiento del bien común. Importante interrogarse también, si estamos desarrollando estrategias pedagógicas en las aulas para el fomento de valores que contribuyan a una visión positiva, constructiva-propositiva y tolerante en los estudiantes, buscando contrarrestar los discursos destructivos que, las burbujas mediáticas, a diario inoculan en las mentes de los jóvenes. Estos son los puntos que de manera general, aborda este escrito, fortalecido de un análisis documental, como primordial método y de la perspectiva del educador que observa y analiza desde el espacio en que interactúa y que nos hace crear posicionamientos de orden práctico que fortalecen las epistemes teóricas. 


\section{¿El docente a la deriva?}

Uno de los argumentos escuchados entre algunos compañeros, tanto dentro como fuera de la Universidad, después de restablecido el orden y la tranquilidad ciudadana en julio del año 2018, es la aparente o real confusión experimentada por docentes durante el fallido intento golpista iniciado el 18 de abril del pasado año. Esta afirmación deja una serie de resquicios de reflexión para nuestras autoridades, gremios, expresiones organizadas de nuestra comunidad universitaria, y de manera particular en cada uno de los académicos, acerca de lo hecho o dejado de hacer en relación a planes e iniciativas de actualización integral del docente universitario y fortalecimiento de su compromiso institucional.

En principio, debemos ser autocríticos y reconocer las debilidades en las políticas de ingreso y/o promoción de docentes a la Universidad. Es cierto que nuestras autoridades han dado pasos importantes en los últimos años, en función de institucionalizar una serie de prácticas y procesos académicos, creando y fortaleciendo normativas, como el ingreso de estudiantes, evaluación al desempeño (puesta en vigencia el pasado 13 de marzo de 2019), movilidad, convenios, entre otros. También, cierto descuido o displicencia durante años, en la labor de formación y actualización docente, ha sido factores que en parte explican el comportamiento de algunos de ellos durante los tres meses que duró la crisis política, así como su actitud posterior. Debe reconocerse como un paso importante en el proceso de mejora institucional, la puesta en vigencia de la "Normativa para la evaluación al desempeño del personal", y como bien lo decía la Maestra Ramona Rodríguez, Rectora de nuestra Universidad, durante el acto de juramentación de las comisiones ad hoc, no tiene carácter punitivo, sino que busca la calidad y el compromiso con la institución.

Dentro de las preocupaciones de mejora institucional, existe conciencia dentro de la educación superior pública, de la herencia histórica sustentada en el modelo napoleónico (Moncada, 2008; 142-143), en su estructura (escuelas-departamentos-Facultad) y enfoque de especializaciones (profesionalizante). Pero también, tenemos fresca en nuestra memoria, el significado de Córdoba de 1918, en cuanto a autonomía y cogobierno, dentro de un concepto "comunitario" de gran trascendencia para la producción de conocimiento y el aporte sustantivo a la sociedad. De igual manera, el Plan de acción (2018-2028) surgido, del encuentro universitario de la III Conferencia Regional de Educación Superior para América Latina y el Caribe (CRES), en ocasión del centenario del "Grito de Córdoba", asumiendo la Agenda2030 para el Desarrollo Sostenible(ODS) promulgada por la Asamblea General de la ONU en 2015, que apunta a la eliminación de la pobreza, hambre cero, igualdad de género, entre otros aspectos esenciales para la paz y el cultivo del bien común.

La concepción de "especialización" del docente actual, no justifica una práctica de "burbuja" (siguiendo a Periser), donde se filtre únicamente lo relacionado a la especificidad del tema que se imparte en el aula de clase. Los contenidos de las asignaturas, sean estas de ingeniería, física, medicina, biología, al igual que las provenientes de las ciencias sociales en general, están inscriptos en entornos sociopolíticos específicos, siendo obligación del académico la puesta en contexto, con la responsabilidad y pertinencia que esta labor requiere. En este sentido, debe preocuparse por el cultivo de un piso cultural integral que le permita ubicar los contenidos de su materia, en su justa dimensión político social, pensando en la misión y visión de la Universidad; práctica esencial y necesaria en nuestro quehacer que posiciona a nuestra Alma Mater (porque somos parte sustantiva de ella) como orientadora y guía de la sociedad.

La Agenda 2030 y los Objetivos de Desarrollo Sostenible (ODS), plantean en su esencia una visión distinta a la actual sociedad. Este documento de la ONU apela a un mundo cuyo sujeto principal es el ser humano, en comunidad, sensible al cuido de la tierra, la solidaridad y la inclusión social. 
En este sentido, durante el análisis y la síntesis de los contenidos temáticos que se hacen en las aulas de clase, no debe perderse de vista esta perspectiva. De manera fundamental, por la posición de docente/investigador apegado a una realidad social concreta, contribuyendo de esta manera a la resolución de los problemas del país e incidiendo en el fortalecimiento del liderazgo.

La postura anterior, pasa por el convencimiento y/o identificación plena y consciente de la visión, misión y objetivos de nuestra Universidad. El principio de universidad pública, abierta a todos los sectores de la sociedad, prioriza a los estudiantes de escasos recursos y comunidades indígenas afro descendientes. Es un punto de partida esencial para asumir la defensa de la institucionalidad del sistema de educación superior con enfoque inclusivo. Los factores golpistas del 18 de abril, tuvieron y tienen en mira la destrucción de la educación pública porque no responde a sus intereses estratégicos de nación si llegan al poder: elitista, plutócrata. Lo ocurrido en nuestra Universidad durante la toma violenta de sus instalaciones, entre el 7 de mayo y el 13 de julio del pasado año, no fue un mero "accidente".

Las campañas de desprestigio continúan, surge la pregunta como docentes ¿Qué estamos haciendo para contrarrestar esta campaña en contra de las Universidades Públicas? Esto requiere una postura ética y moral como académicos, rompiendo con vicios y actitudes complacientes o de mínimo esfuerzo. En la coyuntura actual, debemos --parafraseando a Kennedy-- no preguntar ¿qué puede hacer la Universidad por nosotros?, sino ¿qué podemos hacer por la Universidad?.

\section{Cultura de paz y bien común: cuerpo y alma del discurso docente}

A la llegada al Ejecutivo de la República, del Frente Sandinista de Liberación Nacional (FSLN), por medio de la Alianza Unida Nicaragua Triunfa en 2007 (2011 GRUN), se empezó a escuchar en los discursos oficiales, el concepto de bien común, que hoy día nos es familiar. Es un concepto muy antiguo, que puede encontrarse en "La República" de Platón (como algo que trasciende los bienes particulares) y en "La Política" de Aristóteles (comunidad política que implica buenas acciones para vivir bien). En esta teoría general del Estado de la época antigua, como dice Dieter Nohlen y Rainer Olaf Schultze, et.al. (2006), las normativas y regulaciones sobre el bien común son diversas y hacen referencia a la "vida virtuosa en la comunidad bien ordenada, la idea del derecho, la paz, la libertad, el bienestar y la autorrealización general dentro y por medio de la participación política" (p.158).

Paraautorescomo John Rawls(1979), el bien común, se define como "ciertas condiciones generales que son de ventaja para todos", dentro de un marco jurídico que sustenta las instituciones estatales. Para que el bien común se materialice se requiere de consenso, de tal forma que, proyectos o acciones educativas, productivas y culturales, puedan tomar cuerpo en la vida diaria de las comunidades o grupos sociales. Es un concepto que fomenta la paz fundada, "sobre el principio de justicia y el acatamiento de la ley universal. Si el ordenamiento jurídico logra una verdadera y auténtica paz social, entonces sí, se está realizando el bien común" (Beltranena M, 2010).

La noción de cultura de paz es más reciente, aparece arropado bajo la tutela de la UNESCO a finales de la década de los ochentas del siglo pasado, al crear una Cátedra con ese nombre, apelando a la educación para la paz, la lucha contra la pobreza, el pluralismo cultural y el diálogo intercultural. Es un concepto que ve la educación y sus implicaciones familiares y sociales, como el centro de la convivencia, la tolerancia, sin que eso signifique ausencia de conflicto, estos últimos canalizados en la búsqueda de soluciones colectivas.

Para Isabel Hernández Arteaga (2017), es desde las escuelas y el sistema educativo donde se "construye una cultura de paz". Conceptos complejos por sus implicaciones sociales, porque 
se habla de relaciones entre personas, colectivos con intereses disímiles que interactúan unos a otros y donde las contradicciones afloran. Señala esta autora, que es un esfuerzo multidisciplinar construir una cultura de paz, ya que debe "trascender los límites de los conflictos" [para] convertirse en una vivencia cotidiana de contextos, como la escuela, el trabajo, la familia" (p.152).

De lo anterior, la ley 985, "Ley para una cultura de diálogo, reconciliación, seguridad, trabajo y paz", promulgada por la Asamblea Nacional y aprobada por el Presidente de la República Comandante Daniel Ortega Saavedra, el 24 de enero de 2019, plantea el reto de asumir la tarea desde nuestros espacios de trabajo, familia, escuelas y universidades; resolviendo "nuestros desencuentros, aportando soluciones a la transformación y a la construcción de ambientes saludables, sanos en la familia y la comunidad, mediante estrategias de promoción de valores" (preámbulo de la ley 985).

Ante la situación de violencia política vivida a partir de abril del pasado año, y la fractura social causada por el fallido golpe de Estado que afectó de manera significativa la economía del país y desgarró el tejido familiar y comunitario, al docente universitario se le exige: abandonar cierta actitud pasiva, dejando a un lado su papel de espectador, asumiendo un rol de liderazgo dentro de la comunidad universitaria, en sus diferentes espacios donde interactúa. Por tanto, el docente debe ver la educación -como dice la autora Hernández Arteaga, et. Al (2017), "desde el concepto de paz neutra (...) queactúa desdeel diálogo, busca el consenso (...) y los acuerdos (...) porello que requiere el desarrollo de [un] pensamiento crítico unido a la formación en valores y convivencia" (p.160).

Como se observa, el bien común y el cultivo de una cultura para la paz, convertida en ley de la República por medio del decreto 985, ofrece el marco jurídico de acción y define la política de Estado para los diferentes componentes de la sociedad, de forma que redunde en acciones que fomenten el diálogo, la reconciliación, la armonía, la seguridad ciudadana, y el trabajo. Es una ley que busca en primera instancia, restañar las heridas causadas por el trauma de la violencia extrema que vivió el pueblo nicaragüense entre abril y julio del pasado año, sentando las bases de una nueva cultura de convivencia y concordia. Como docentes, debemos de apropiarnos de los ejes, actores y acciones que la ley señala e incorporarlos como un componente esencial de nuestros contenidos y apoyo intrínseco de la metodología de enseñanza en nuestra aula de clase. De esta manera se puede construir una pedagogía social (Rojano Mercado: 2008; 39; Freire; 1983) para la paz y la convivencia desde la Universidad.

\section{Re-pensando estrategias pedagógicas en valores}

La experiencia vivida en Nicaragua, Venezuela y otros países, agredidos por el imperialismo norteamericano, utilizando las redes sociales, como una nueva arma de dominación, y las posibilidades que el uso tecnológico de los algoritmos encierra, en la llamada guerra de quinta generación, ha puesto en agenda el tema de los valores, éticos y morales, sustento clave para caracterizar y/o juzgar el comportamiento individual y colectivo de las personas. En este sentido, durante el siglo XXI en que nos encontramos, debemos comprender que las "armas de destrucción masiva" (término militar en uso durante la retórica de guerra fría) no son aquellas que desde el punto de vista material, destruyen los hábitats humanos, sino las que de manera furtiva o subliminal, penetran la siquis de las personas, exterminando sentimientos nobles al pulverizar las estructuras de pensamiento, en medio de un cuadro desolador de caos que facilita la dominación imperial u otras causas extremas.

La batalla por "las mentes y corazones", iniciada con el boom del internet y las redes sociales, conlleva una serie de implicaciones que de manera transversal, cruzan la vida de las personas y las colectividades. En apariencia, se presenta como una lucha pacífica, pero de 
manera implícita contiene una carga de violencia y destrucción acumulada, similar al efecto de una "bomba de hidrógeno". La experiencia de abril de 2018, es un ejemplo del trabajo silencioso, sistemático y perseverante para la creación de un frente de guerra mediático.

El uso y consumo de las redes sociales de manera irrestricta por grupos opositores, la tolerancia gubernamental (en extremo permisible) de la circulación de mensajes tendenciosos y manipulados, producidos desde medios convencionales y/o alternativos de comunicación, crean en poco más de una década, el escenario político ideal de violencia destructiva e irracional contra la institucionalidad. Narcotizados por el sistemático consumo de mensajes tóxicos, los agentes de este "ejército cibernético" pasan a las agresiones físicas en abril 18 del 2018 (semejante a una cábala diabólica) denotando un cuadro de enajenación y paroxismo inusitado, al protagonizar de manera real una serie de puesta en escena que imitan (recuerdan) a Yo Robot (Proyas, A; 2004) y a Terminator 2: el juicio final (Cameron, J.; 1991). Es la consecuencia de grupos domesticados, atrapados bajo las redes de los filtros burbujas que bien explica Eli Periser (2017) en su libro El Filtro Burbuja, "Cómo la red decide lo que leemos y lo que pensamos".

Estratagema aplicada a partir de experiencias vividas en otros países (primavera árabe), y de las recetas de ideólogos del imperialismo (Gene Sharps) donde una vasta estructura mediática de uso en la actualidad, sirve de plataforma para las agresiones, la destrucción y la dominación, en el contexto de la guerra de quinta generación que vivimos. Detrás de los trolls que fabrican noticas falsas (fakes news) buscando vulnerar valores y creencias de los usuarios de las redes sociales y medios de comunicación electrónica, está todo un sistema bélico mediático controlado por los halcones del Pentágono. El objetivo político de los ejecutores del plan político de abril del pasado año era: acabar con la institucionalidad del país, socavando los valores y principios históricos y culturales que sustenta el imaginario social, y familiar del nicaragüense.

Larealidad arriba descrita, sugiere quetodos los sectoressociales, amantes de la paz, la convivencia pacífica y la solidaridad en pro del bien común, deben asumir una posición que contribuya a la solución de los problemas y no ser parte de ellos. Desde la posición del docente universitario, implica la estructuración de una pedagogía que recupere el sentido constructivo de los valores de convivencia en comunidad, se promueva la tolerancia y el respeto a la diversidad de ideas, vulnerados por la violencia esquizofrénica emprendida por factores políticos sedientos de poder.

Tarea nada fácil, si analizamos los instrumentos utilizados por los dirigentes de esta guerra de quinta generación. La colonización de la mente y conciencia de las personas con fines de instrumentalización, es el primer paso para el reclutamiento de los que están en la primera línea de "combate" en esta contienda mediática. La destrucción moral y física de colectividades o grupos sociales es el segundo, no importando relaciones de afinidad, consanguinidad o afectividad; dejando a las personas vacías, huecas en su sentido de humanidad, objetivo último a alcanzar en el campo de batalla cibernético.

Un claro ejemplo de la degradación humana por medio de las redes sociales y su consumo sin reparo alguno (sin que medien estrategias para la utilización sana y constructiva), es la actitud violenta, agresiva y escéptica de personas que, por diversas razones, se vieron arrastradas por la vorágine de la violencia mediática orquestada por agentes especialistas, bajo las órdenes de políticos extremistas. Algunas de estas personas alienadas, niegan sin rubor alguno, la destrucción de la Universidad durante el período de la toma por la fuerza del campus (abril-julio de 2018). Es más, llegan al cinismo paranoico de afirmar que fue una autodestrucción. Aventurando un paralelismo extremo, esta actitud nos recuerda la postura de células neo nazi europeas que niegan el holocausto perpetrado en contra de la comunidad judía durante la Segunda Guerra Mundial. 
La literatura señala que el valor ético está ligado de manera intrínseca con la individualidad, y la moral con la sociedad (Ortiz Millán, 2016; 119-125), ambas afectadas por la crisis política que enfrentamos desde el 2018. Encontrar el equilibrio que restañe la confianza y promueva el diálogo, como una forma de respeto y convivencia, es una tarea urgente que en estos momentos de desgarro del tejido social, el docente universitario no debe soslayar. Eso implica, la formulación de una pedagogía que sobre la base del quehacer integral del día a día de los estudiantes, amigos, y familiares, revitalice los contenidos de los conceptos de autoridad, autonomía, confianza, amistad y solidaridad. De esta manera, la articulación de los discursos individuales y colectivos y la práctica de vida, exigen renovación a partir de la reapropiación y/o re significación de estas estructuras de pensamiento.

En otras palabras, debemos pensar en la experiencia de una pedagogía que retome el espíritu que motivó a Paulo Freire $(1980 ; 1983)$ en la década del 60 y 70 del siglo pasado, al apropiarse de la persona (en su carácter de sujeto social) como objeto de su estudio, enmarcado en su entorno cotidiano e inmerso en sus complejas relaciones sociales. Con sus motivaciones, problemas, se erigen en sujeto de su propia liberación. Estrategia que pasa por la autoconciencia de la realidad circundante y las posibilidades de enfrentarlas sin arredrarse. Tal es el caso nicaragüense, donde las desigualdades representan retos estructurales; los poderes fácticos (religiosos y de clase) y mediáticos, se resisten a sujetarse y/o respetar la legalidad institucional; y mezquinos grupos económicos y financieros (vinculados a esos poderes fácticos), defienden como sagradas las leyes que sustentan el modelo capitalista.

\section{Conclusión}

Las crisis políticas en cualquier contexto, por sus implicaciones o connotaciones en todo el tejido social, actores económicos y culturales, no deja indiferente a nadie. Los hechos ocurridos a partir de abril de 2018 demostraron contradicciones que creíamos superadas, entre ellas la violencia como instrumento para dirimir las controversias. De igual manera, puso en la agenda pública, la necesidad de trabajar en temas que atiendan y/o prioricen la comunicación con todos los sectores sociales y económicos, mejorando los canales de participación individual y colectiva, en sus comunidades, territorios, colectivos de trabajo, pequeños, medianos o grandes emprendimientos, en función de la atención y resolución de los problemas de cada una de sus expresiones organizadas.

Consensuar una agenda pública de comunicación (siguiendo el espíritu de Paulo Freire de coparticipación) como canal indispensable para atender y emprender iniciativas o demandas de los diversos sectores de la sociedad. Por su forma organizativa, beligerancia y actores implicados, los sucesos de abril de 2018, evidenciaron que el cultivo del diálogo como mecanismo civilizado para la resolución de controversias, requiere de mayores compromisos, consensos y mayor integración. La campaña de reconciliación emprendida por el gobierno desde el 2007 es una iniciativa importante que debe relanzarse y reactualizarse, desde las diversas instituciones públicas, gremiales o cooperativas.

A partir de la complejidad del análisis de la situación que llevó a la violencia del 2018, las universidades públicas, entre ellas la UNAN-Managua, por su esencia social y cultural, son actores de gran importancia en la búsqueda de la superación de la crisis política que vive el país. Desde sus inicios, las autoridades superiores universitarias han estado al frente de los 
requerimientos de los diversos sectores sociales, llamando al diálogo, la paz, la reconciliación; incorporándose a comisiones inter estatales u otras iniciativas públicas (Más de una docena de comunicados desde abril hasta agosto de 2018 respaldan esta posición).

La beligerancia de nuestra Universidad para contribuir a la resolución de la crisis por medio del diálogo, ha tenido su correlato a lo interno de las estructuras de nuestra Alma Mater. Los talleres de Convivencia iniciados en agosto de 2018 apuntaban a fomentar el diálogo y comunicación asertiva, coherente con la preocupación de nuestro rectorado de preparar a nuestra comunidad universitaria para enfrentar la crisis, tanto en nuestro entorno laboral, las aulas de clase y en las relaciones de extensión e internacionalización que por función promueve esta casa de estudios superiores.

Las preguntas que estructuran este trabajo, aluden a la contribución del docente en la resolución de la crisis. Si bien es cierto, nuestras autoridades están al frente de los esfuerzos e iniciativas nacionales, inter institucionales, también; que los docentes en particular debemos contribuir, de manera individual o colectiva (por medio de equipos de trabajo, gremios, etc.), en nuestras aulas de clase, familiares, amigos, espacios académicos nacionales o internacionales, en la creación de un clima de diálogo, tolerancia y respeto a las diferencias. Sin embargo, la experiencia diaria no sugiere entusiasmo o motivación suficiente entre docentes para asumir un liderazgo fuerte y coherente, desde nuestras disciplinas y asignaturas, en la promoción, orientación y aclaración a nuestros estudiantes, acerca del fomento de los valores arriba indicados.

De lo anterior, otra pregunta que se aborda de manera general en este trabajo, es la perentoria necesidad de apropiarse (algunos docentes lo hacen suyo de manera consciente o inconsciente), de la cultura de paz y su correlato, el bien común como componente esencial en la dinámica pedagógica docente. Dos conceptos que están presente en la ley 985 "Ley para una cultura de diálogo, reconciliación, seguridad, trabajo y paz", que respalda iniciativas en todos los ámbitos de la vida nacional, en función de una cultura incluyente, propositiva e innovadora, coherente con las políticas que nuestra Universidad y sus autoridades promueven. Como inquietud válida en estas circunstancias ¿Estamos los docentes a la altura de estas exigencias?

Por último, debemos pensar en una pedagogía que de manera transversal incorpore los contenidos de cultura de paz y bien común en nuestro quehacer universitario. Una condición indispensable que haga realidad esta práctica educativa, pasa por despojarnos de prejuicios y antivalores que nos aprisionan, arraigados en nuestra dinámica académica, entre ellos los "feudos" o intereses de grupos. Esto implica, apropiarse y poner en práctica valores sustentados en la misión y visión de la UNAN-Managua, siendo consciente del lugar que ocupamos en la estructura social del país, como guías y formadores de profesionales que serán referentes en sus entornos sociales, laborales y culturales. 


\section{Referencias bibliográficas}

- $\quad$ Beltranena de Padilla M, L (2010); que se entiende por el bien común. UNIVERSIDAD FRANCISCO DE MARROQUIN, Facultad de Derecho, Guatemala. En https://derecho.ufm. edu/que-se-entiende-por-el-bien-comun/).

- Freire, P. (1983) Extensión o comunicación. La concientización en el medio rural. México: Siglo XXI Editores.

- Hernández Arteaga, I. et.al (enero-junio de 2017). Cultura de paz: Una construcción educativa aporte teórico. Revista Historia de la Educación Latinoamericana. 19 (28). Pp.149-172.

- $\quad$ Ley N 985. La Gaceta. Diario Oficial. Managua, Nicaragua, 28 de enero de 2019.

- Moncada, J.S. (agosto de 2008) La Universidad. Un acercamiento histórico-filosófico. Ideas y Valores, (137), Pp. 131-148)

- Nohlen, D. y Schultze, R-O et al. (2006). Diccionario de Ciencia Política. Teorías, métodos, conceptos. México: Porrúa/El Colegio de Veracruz.

- Ortiz Millán, G. (octubre 2016) sobre la distinción entre ética y moral. ISONOMIA, (45), Pp. 119-125.

- Pariser, E. (2017) El Filtro Burbuja. Cómo la red decide lo que leemos y lo que pensamos. Barcelona: Taurus.

- Rawls, J. (1979). Teoría de la justicia. México: Fondo de Cultura Económica.

Dr. José Miguel Ayerdis García.

Dr. En ciencias históricas, catedrático universitario, autor de distintos artículos científicos en revistas nacionales e internacionales, actualmente Director de la Dirección de Relaciones Internacionales de la UNAN-Managua. https://orcid.org/0000-0001-9677-7786 mayermis@unan.edu.ni 